The Neuroscience of Decision Making and Our Standards for Assessing Competence to Consent

Steve Clarke

Oxford Martin Institute and Faculty of Philosophy

University of Oxford

Stephen.Clarke@philosophy.ox.ac.uk

Tel: 01865-286979

Fax: 01865-286983

\begin{abstract}
Rapid advances in neuroscience may enable us to identify the neural correlates of ordinary decision making. Such knowledge opens up the possibility of acquiring highly accurate information about people's competence to consent to medical procedures and to participate in medical research. Currently we are unable to determine competence to consent with accuracy and we make a number of unrealistic practical assumptions to deal with our ignorance. Here I argue that if we are able to detect competence to consent and if we are able to develop a reliable neural test of competence to consent, then these assumptions will have to be rejected. I also consider and reject three lines of argument that might be developed by a defender of the status quo in order to protect our current practices regarding judgments of competence in the face of the availability of information about the neural correlates of ordinary human decision making.
\end{abstract}

Keywords

Competence; Decision Making Capacity; Informed Consent; Neural Correlates; Rule of Thumb; Status Quo 
The Neuroscience of Decision Making and Our Standards for Assessing Competence to Consent

\begin{abstract}
Rapid advances in neuroscience may enable us to identify the neural correlates of ordinary decision making. Such knowledge opens up the possibility of acquiring highly accurate information about people's competence to consent to medical procedures and to participate in medical research. Currently we are unable to determine competence to consent with accuracy and we make a number of unrealistic practical assumptions to deal with our ignorance. Here I argue that if we are able to detect competence to consent and if we are able to develop a reliable neural test of competence to consent, then these assumptions will have to be rejected. I also consider and reject three lines of argument that might be developed by a defender of the status quo in order to protect our current practices regarding judgments of competence in the face of the availability of information about the neural correlates of ordinary human decision making.
\end{abstract}

Keywords

Competence, Decision Making Capacity, Informed Consent, Neural Correlates, Rule of Thumb, Status Quo 


\section{The Neuroscience of Decision Making and Our Standards for Assessing Competence to Consent}

\section{Competence to Provide Informed Consent}

In order to provide ethically and legally valid informed consent to undergo a medical procedure or to participate in medical research, a patient, or prospective research subject must be competent to do so [1]. Competence is a threshold concept. All of those who are judged to be competent to drive a car are judged to be able to drive a car at or above a certain level of ability, and are hence considered eligible to receive a driver's licence. Those who fail to demonstrate an ability to meet this threshold of competence are generally considered ineligible to receive a driver's licence. Although competence is a threshold concept it would be a mistake to suppose that there is an absolute threshold between competent and incompetent drivers out there in the world waiting to be discovered. There is a spectrum of different levels of driving ability and a society chooses where to set the threshold between competent and incompetent drivers on this spectrum.

A society may choose to set the threshold between competent and incompetent drivers at a variety of different levels of driving ability depending on the goals that it wishes to promote. If we wish to promote safety we might choose to set the threshold at a high level, whereas if we wished to promote car ownership and use, and are willing to tolerate an increased number of accidents we may choose to set the threshold lower than we would otherwise. ${ }^{1}$ Possession of a driver's licence is an indication of driving competence. It is an indication that licensed drivers are competent to drive in many circumstances, but it is not an indication that they are

\footnotetext{
${ }^{1}$ However, if we set the threshold too low we might fail to promote car ownership and use as the resulting increased chance of being involved in a driving accident may deter people who are sufficiently risk averse from owning and using cars.
} 
competent to drive under all circumstances. Otherwise competent drivers are typically not judged to be competent to drive when they are inebriated, severely sleep deprived, suffering from an injury that restricts their ability to control a car's steering wheel, and so on.

The threshold we set between competence and incompetence to consent is determined by the underlying capacities that enable competent informed consent to be provided. The key capacity underpinning competence to consent is generally agreed be the capacity to make rational decisions [2-3]. An influential analysis of rational decision making capacity (DMC) is due to Welie and Welie, who suggest that this involves: (i) the ability to absorb, comprehend and retain knowledge, or more broadly, cognitive content; (ii) the ability to manipulate cognitive content critically; (iii) freedom of will; and (iv) the ability to express oneself [4]. Like driving, decision making for the purpose of informed consent requires the integration of a number of skills. However, unlike driving, there is no agreed upon test of competence to provide informed consent. A variety of instruments are sometimes used to test the presence of the DMC that underpins competence to consent, including the MacArthur Competence Assessment Tool for Clinical Research, the California Scale of Appreciation and the Deaconess Informed Consent Comprehension Test [5-7]. None of these instruments has obtained widespread acceptance and there is a distinct lack of evidence of the reliability and validity of all of them [8].

Medical professionals do not generally rely on tests to determine competence to consent in patients and nor do researchers for research subjects. Ordinary people are assumed to be competent to consent and tests are only administered if there is some serious doubt as to the competence of particular individuals. In part this assumption is made because the instruments to determine competence are not known to be reliable and in part because medical professionals are typically charged with a responsibility to determine competence to consent 
and most medical professionals have no particular expertise in identifying the relevant decision making capacity [9]. In part this assumption is also made because the threshold of competence that we choose is usually set relative to the abilities of cognitively typical people in our society and is designed to ensure that the great majority of those who are cognitively typical are judged competent to provide informed consent [10]. It is set this way because in Western societies we are particularly concerned to uphold the value of individual autonomy. We generally think that it is important that people are able to decide for themselves how to live their own lives and interpersonal relations in Western societies are typically structured around the ideal of mutual consent [11]. Medical practice had been something of an exception to this generalisation. Until the last quarter of the Twentieth Century, medicine was dominated by a culture of paternalism. Nowadays, however, in Western societies medicine has fallen into line with the rest of Western culture, at least ideologically, and has taken significant steps to shake off its paternalistic cultural past and to embrace a culture of respect for autonomy, which is, in large part, why informed consent is sought from patients and medical research subjects [12].

Whenever a medical professional is called upon to make a determination regarding the competence of a patient or a prospective medical research subject to provide informed consent (relative to the standards of DMC that are taken to be sufficient to enable informed consent to be provided in a given society) she is faced with the possibility of making two different types of error. She may decide that a patient is competent to provide informed consent, when that patient is not competent to provide informed consent (a false positive) or she may decide that a patient is not competent to provide informed consent when that patient is competent to provide informed consent (a false negative). Because our current means of identifying competence to consent are not very accurate we are in danger of being mistaken a significantly large number of times. However, we can choose how to distribute these errors. If we are strict in applying our standards of DMC before accepting that 
someone is competent to consent we will avoid most false positives, but at the cost of allowing many false negatives. If we apply lenient standards, then we will avoid most false negatives, but at the cost of allowing many false positives. In practice, at least in Western societies, we take the latter course of action. We take the view that it is much better to allow people who are not competent to consent to be judged competent than to deprive those who are competent to consent of the right to determine whether or not they are going to undergo a medical procedure or participate in medical research. This is because, as we saw, we place a very high value on individual autonomy. We would regard it as outrageous if someone was forced to undergo a medical procedure or participate in medical research against their will on the mere suspicion that they are not competent to consent. But we do not regard it as similarly outrageous if someone is allowed to determine whether or not to undergo an operation or participate in medical research when there is some doubt as to their competence to make such a determination.

It is difficult and time consuming for medical professionals to determine competence to provide informed consent to even a reasonable degree of accuracy. In practice medical professionals typically proceed by applying a number of rules and rules of thumb which enable them to avoid having to make individual determinations regarding competence to provide informed consent in many cases. One such rule is that the DMC needed to provide valid informed consent is not present before a particular age and generally is present after that age threshold is reached. This threshold varies from country to country (in the UK it is 16) but is generally thought to be reached sometime during an individual's teenage years. ${ }^{2}$

\footnotetext{
${ }^{2}$ In the UK this is very much a rule of thumb. The default age of consent is 16 , however, a child under the age of 16 may be deemed to be competent to consent if they are judged to be mature enough to understand the nature and implications of undertaking a medical procedure. This is known as 'Gillick competency.' For more on Gillick competency see [13].
} 
A second rule of thumb is that people's levels of competence to consent do not generally vary across different circumstances; or at least they do not generally vary in such a way that the same person is found on different sides of the threshold of competence in many different circumstances. We only allow that competence to consent will shift from one side of the threshold to the other under extreme changes in circumstances. For example, if a patient who is generally considered to be competent to provide informed consent is found to be drunk then we will not allow them to provide informed consent. Instead we will typically insist that they wait until the period of inebriation is over and they have 'sobered up' before they are allowed to provide informed consent. In the main, however, we make a one off determination of competence or incompetence and avoid having to update this determination in response to variations in circumstances, unless it becomes very clear that this determination requires updating.

A third rule of thumb that doctors often apply is that if a patient is competent to provide informed consent to one medical procedure then they are competent to provide informed consent to any other medical procedure. Mutatis mutandis for participation in medical research. Doctors typically make this assumption despite knowing that some decisions that a patient or research subject may be required to make are much more complicated than other decisions. In order to provide valid informed consent to many forms of cardiac surgery a patient will be required to comprehend a complicated procedure, including post operative medical care, as well as appreciating the various risks involved in the procedure. Furthermore, they may be required to comprehend the details of alternatives to the surgery, which may also be very complicated. By contrast, arthroscopic surgical procedures on knees are typically simple affairs with few risks involved. It is much more cognitively demanding to provide valid informed consent to complex cardiac surgery than it is to provide informed consent to routine arthroscopic surgery on one's knee. Nevertheless it is generally assumed that if a patient is competent to consent to one of these then they are also competent to consent to the other. 
This third rule of thumb is perhaps the most controversial of the three, and there have been calls in the medical ethics literature - notably by James Drane [14] - for doctors to apply a sliding scale of competency according to the difficulties involved in patient decision making regarding more or less complicated medical procedures. However, this suggestion has not generally been adopted, in large part because it would be very impractical to implement. Doctors find it hard enough to apply a threshold of competence consistently. Applying a sliding scale of competence and doing so consistently would require doctors to acquire significant expertise in understanding the relationship between DMC and the difficulty of decision making for the purposes of providing informed consent to different procedures. And implementing a sliding scale of competency would raise further practical difficulties [15].

One further practical difficulty that would be raised by implementing a sliding scale of competency, is that when a medical procedure does not go as anticipated, it may unexpectedly become a more complicated procedure. It seems deeply impractical to allow for the possibility that a patient can start out as competent to consent to a procedure, but end up as incompetent to consent to that procedure when unanticipated complications emerge. Another problem is that it is common for a patient to seek a second expert opinion when contemplating a recommended medical procedure. Doctors may well disagree about the exact nature of a particular patient's condition and may thus suggest different solutions. This raises the possibility that a particular patient may be considered competent to consent to undergo a course of treatment that one doctor recommends, but incompetent to consent to undergo a course of treatment that another doctor recommends. But this possibility undermines a major purpose of patient's seeking of second opinions, which is to widen the choice of options available to them. For practical reasons such as the above doctors generally set aside the 
possibility that a patient can be competent to consent to some medical procedures but not competent to consent to others $[15]^{3}$

\section{Neuroscience and Informed Consent}

Rapid progress in neuroscience is enabling us to begin to track the neural correlates of decision formation as it is implemented in the human brain [17]. We are beginning to understand, inter alia, what goes on in our brains when we decide to act, [18-19] the role of emotions in decision making as manifested in the brain, [20-21] and the role of subjective values in decision making as manifested in the brain [22-23]. If such progress continues then it seems reasonable to think that we may be able to identify the neural correlates of decision making for the purposes of informed consent in the not-too-distant future, a possibility that has already been raised by several authors [24-26]. This knowledge may enable us to develop highly accurate tests of the presence of the DMC necessary to enable the provision of valid informed consent. Here I consider what may happen to our informed consent practices if advances in neuroscience lead to us developing a highly accurate neural test of competence to consent that is easy to conduct and not overly expensive. All of this may happen and its consequences will be profound if it does happen so it is important that we try to consider what these consequences will be. We should try to plan for plausibly possible future scenarios in which technology challenges our social practices, in so far as we can [27].

\footnotetext{
${ }^{3}$ Similar issues arise in legal contexts. It seems plausible to think that defendants who face multiple charges might have sufficient mental capacity to be competent to stand trial to some of these but not others. However, courts have generally been reluctant to allow for this possibility, on pragmatic grounds [16].
} 
One consequence of having an accurate test of competence to provide informed consent would be many less instances in which we were uncertain whether or not particular patients and medical research subjects were competent to provide informed consent. Provided that our standards for competence to consent remained unchanged then we would almost certainly end up considering many more people not to be competent to provide informed consent than we do now. This is because, as we have seen, we currently err on the side of caution and in the overwhelming majority of cases of doubt we allow that people are competent to consent. If our test of competence was highly accurate it is reasonable to conclude that very many cases of doubt that are currently treated as cases of competence would be shown to be cases of incompetence, so we would end up refusing to allow nearly as many people to provide informed consent as we do now (assuming our standards remain unchanged).

Another consequence is that our various rules and rules of thumb, which we use to simplify the procedure of deciding whether people are competent to provide informed consent or not, would be shown to be unrealistic and would therefore, be untenable. It would be extremely difficult to continue to apply the rule that people are generally able to provide valid informed consent at age 16 , say, if neuroscientific advances revealed that many of them are competent to provide informed consent at younger ages and many others are not competent to provide informed consent until they are significantly older.

Similarly, it would be difficult to continue to allow people to provide informed consent across a wide range of circumstances if neuroscientific advances revealed that peoples' ability to provide valid informed consent is significantly diminished by a wide range of circumstantial factors. If, for example, we established that overeating, physical exhaustion, bereavement, being in the throes of romantic love, being overly stressed and/or 
being depressed, ${ }^{4}$ all tend to lead to a significant impairment of peoples' competence to provide informed consent, then we face a choice between introducing many more qualifications to our rule of thumb to exclude people from providing informed consent under particular circumstances, or ceasing to use this rule of thumb and directly examining the neural correlates of competence to provide informed consent whenever people's circumstances varied significantly.

The rule of thumb that, if people are competent to consent to any one medical procedure or operation then they are competent to consent to any other would also be very difficult to maintain. If we can reliably establish that many people are competent to consent to some operations that are easy to comprehend but not others that are more difficult to comprehend - and it seems plausible to think that we may be able to establish exactly this then it would become very difficult to maintain the fiction of consistency in their ability to provide informed consent. Trying to maintain such a fiction would lead us to insist that people who were competent to consent to relatively simple medical procedures were also invariably competent to consent to complicated procedures. But it would be extremely difficult to insist on this generalisation if we had good reason to suspect that they were not competent to consent to complicated medical procedures and if we also had the means to determine that they were not competent to consent to complicated medical procedures.

\section{Defending the Status Quo?}

I have argued that if advances in neuroscience lead us to develop a highly accurate neural test of competence to consent then a number of our current practices regarding informed consent will need to change. How might

\footnotetext{
${ }^{4}$ In a recent paper Jotterand et al. deploy neuroscientific results to argue that competence to provide informed consent is impaired by treatment resistant depression [28].
} 
someone who disputed this claim and wanted to argue that our current practices could and should remain as they are even if neural testing becomes available? Here I consider three possible lines of argument for the conclusion that such advances are compatible with our current practices. These are (i) an appeal to cognitive privacy, (ii) an argument for lowering the threshold for competence to consent to compensate for the anticipated changes and (iii) an argument for improving peoples' competence to consent where it is deficient, to compensate for anticipated changes. I will argue that while all of these lines of argument raise important considerations, none of them is a suitable basis for a defence of the claim that our current practices are compatible with the anticipated advances.

\section{(i) Cognitive privacy}

I have considered the implications of making information about the neural states of patients available to medical professionals for the purposes of determining competence to consent. But it might be argued that such neural tests should not, and therefore would not be used as their use involves a straightforward violation of cognitive privacy. ${ }^{5}$ There are many good reasons to value cognitive privacy, including a legitimate desire to be able to present ourselves in different ways in different circumstances [30-31]. The range of the right to privacy is much disputed [32]; but if there is any right to privacy at all, then it seems that the right to the privacy of our most intimate thoughts falls within the range of that more general right.

It might be supposed that a right to cognitive privacy would be incompatible with the use of neuroscientific techniques to detect the neural correlates of DMC for the purposes of informed consent. However, we should bear in mind that private information, including very sensitive information, is often disclosed by patients and

\footnotetext{
${ }^{5}$ Farah et al. have recently argued that concerns about violations of brain privacy are realistic [29].
} 
research subjects to medical professionals. For example, details of a patient's sexual history will usually be disclosed to a doctor treating venereal disease. These disclosures have a minimal impact on a patient and research subject privacy when they are provided with a credible undertaking of patient or research subject confidentiality, which, in large part explains why patients and research subjects agree to make them. Typically, concerns about privacy in this context are concerns that personal information will enter the public sphere.

Someone who might be mortified to have the details of their sexual history discussed in public is nevertheless likely to consent to have it disclosed to a doctor or medical researcher if and when she has reason to trust that the disclosure will remain confidential. ${ }^{6}$ It seems then that the use of neuroscientific techniques to identify the neural correlates of DMC for the purposes of providing informed consent is compatible with respect for the value of cognitive privacy if the norm of patient and research subject confidentiality is upheld. So this line of objection is not an effective defence of the status quo in the face of the technologically driven changes that we are contemplating.

(ii) A lowered threshold

As we saw earlier the level at which we set the threshold of DMC required to enable valid informed consent to be provided can be varied. If, as I have argued, a consequence of having an accurate test of the neural correlates of DMC for the purposes of informed consent is that less people are considered able to meet that threshold than when our testing procedures were less accurate, then one response to this new state of affairs is that we should try to correct for this change by lowering our threshold of competence.

\footnotetext{
${ }^{6}$ While the norm of respect for patient confidentiality is generally upheld in medical practice more needs to be done before the same can be said about the norm of respect for research subject privacy in medical research [33].
} 
It is true that we could lower the threshold of competence required for informed consent for particular medical procedures and by doing so ensure that the overall proportion of the population who are considered competent to provide informed consent remains (roughly) unchanged. It is far from clear that that we should do this. In the absence of a good reason to suppose that the status quo happens to be at the right place we have reason to suspect that the advocate of this policy response is in the grip of 'status quo bias'. ${ }^{7}$ But even if we were to lower the threshold of competence to provide informed consent this would not provide any basis for retaining the three rules and rules of thumb that we currently use to determine competence to provide informed consent and which were mentioned earlier. There is no reason to think that the distribution of patients and prospective medical research subjects who meet a lowered threshold would match the current distribution in the absence of our rules of thumb being applied. We might end up with the same overall proportion of the population being considered competent to provide informed consent, however, our new distribution would include some people under the current age of consent and exclude some people over the current age of consent. It would also exclude people in circumstances that we do not currently exclude people from providing informed consent and it would include significant numbers of people who were only able to consent to simple medical procedures and not to complicated ones, a combination that is very rare today given the use of our rules and rules of thumb.

(iii) Improving People's Competence to Consent.

The final argument for retaining the status quo is that advances in neuroscience can be expected to occur on a number of fronts. In seems reasonable to suppose that when we are at the stage of being able to detect the neural correlates of DMC for the purposes of providing informed consent, we will also be able to intervene in such a way as to correct peoples' cognitive deficiencies and will thereby be able to intervene to retain the status quo.

\footnotetext{
${ }^{7}$ For discussion of status quo bias and other default biases see [34].
} 
Unlike the appeal to a lowered threshold this could potentially allow us to retain the utility of our current rules and rules of thumb. We could, if we chose, intervene in such a way as to ensure that the current rules and rules of thumb accurately matched the distribution of competence to provide informed consent. For example, if our current rule is that competence to provide informed consent is acquired at age 16 then we could perhaps intervene in the brains of those who attain an age of 16 , but who have yet to acquire the capacity to provide informed consent, and increase their DMC.

This suggestion faces several objections. One objection is that the assumption that progress in detecting the neural correlates of DMC for the purposes of providing informed consent will be matched by progress in developing the capacity to improve DMC may be a mistaken one. It may also turn out that we are able to develop the means to reliably detect the neural correlates of DMC for the purposes of providing informed consent much earlier than we acquire the means to intervene to significantly improve DMC. A second objection is that, by themselves, such interventions would not do enough to maintain the status quo. Under the status quo those who do not meet the required age threshold to provide informed consent are generally considered incompetent to provide informed consent, however examination of the neural correlates of their DMC for the purposes of providing informed consent may reveal that they are competent to provide informed consent to at least some medical procedures. Unless we also intervened to reduce their DMC them then we would not succeed in retaining the status quo, but it seems deeply implausible to think that people would accept interventions that reduced DMC, especially in children. A third objection is that if we have the ability to improve DMC then there is not much reason to think that we would choose to use this ability just to uphold the status quo. If we could, we might choose, for example, to ensure that every last adult has sufficient cognitive capacity to make competent decisions for the purposes of providing informed consent to all medical procedures. 
Having the ability to increase DMC opens up the possibility of cognitive enhancement, which would, in this context, involve increasing DMC above levels that humans are ordinarily able to obtain. ${ }^{8}$ If most of us were cognitively enhanced and were highly competent to provide informed consent then it might be that we would choose to raise our threshold of competence to provide informed consent, making it much harder for the few who were not cognitively enhanced to provide competent informed consent. Much has been written about the ethics of enhancement [36-37]. I do not want to enter into these debates here. I simply note that a defender of the status quo with regard to informed consent who appealed to this final line of argument would need to offer convincing arguments against the enhancement of DMC at least for the purposes of enabling informed consent.

\section{Concluding Remarks}

I have argued that advances in neuroscience that may enable us to identify the neural correlates of ordinary decision making will have profound consequences for our practices regarding the determination of competence to provide informed consent. The availability of an accurate, affordable test of the DMC for the purposes of providing informed consent would have a significant effect on the overall number of people who are judged to be competent to provide informed consent to undergo a medical procedure or to participate in medical research and would lead us to abandon several rules and rules of thumb that we currently use to determine competence to provide informed consent. I went on to consider three ways in which a defender of the status quo in the face of

\footnotetext{
${ }^{8}$ For discussion of the prospects of future cognitive enhancement and related ethics and regulatory issues see [35].
} 
neurological advance might argue that such technological advances could be shown to be compatible with our current practices. However, none of these appeared to be successful. ${ }^{9}$

\section{References}

1. Beauchamp, T. L. and Childress. J. F. 2001. Principles of Biomedical Ethics (5th Ed.). New York: Oxford University Press: 79.

2. Buchanan, A. and Brock. D. 1990. Deciding for Others: the Ethics of Surrogate Decision-Making. Cambridge: Cambridge University Press: 18.

3. Gert, B. Culver, C. M. and Danner Clouser, K. 1997. Bioethics: A Return to Fundamentals. New York: Oxford University Press: 137.

4. Welie, J. V. M. and Welie, S. P. K. 2001. Patient Decision-Making Competence: Outline of a Conceptual Analysis. Medicine, Health Care and Philosophy 4: 127-138: 133.

5. Appelbaum, P. S. and Grisso, T. 2001. MacCAT-CR: MacArthur Competence Assessment Tool for Clinical Research. Sarasota, FLA: Professional Resources Press.

6. Saks, E. R., Dunn, L. B., Marshall, B. J., Nayak, G. V., Goldshan, S. and Jeste, D. V. 2002. The California Scale of Appreciation: A New Instrument to Measure the Appreciation Component of Capacity to Consent to Research. American Journal of Geriatric Surgery 10: 166-174.

7. Miller, C. K. O’Donnell, D. Searight, S. and Barbarash. R. A. 1996. The Deaconess Informed Consent Comprehension Test: An Assessment Tool for Clinical Research Subjects, Pharmacotherapy 16: 872878.

\footnotetext{
${ }^{9}$ Thanks to an anonymous reviewer as well as audiences at the Philosophy Department, University of South Carolina, the Centre for Applied Philosophy and Public Ethics, Canberra Division, and the 10th World Congress of Bioethics, Singapore 2010 for helpful comments on earlier drafts of this paper.
} 
8. Dunn, L. B., Nowrangi, M. A., Palmer, B. W. Jeste D. J. and Saks E. R.. 2006. Assessing Decisional Capacity for Clinical Research or Treatment: A Review of Instruments, American Journal of Psychiatry 163: 8: 1323-1334.

9. Appelbaum, P. S. 2007. Assessment of Patients' Competence to Consent to Treatment, New England Journal of Medicine 357: 1834-1840: 1835.

10. Iltis, A. 2006. Lay Concepts in Informed Consent to Biomedical Research: The Capacity to Understand and Appreciate Risk. Bioethics 20: 180-190.

11. Kleinig. J. 1982. The Ethics of Consent. In New Essays in Ethics and Public Policy. In K. Nielsen and S.C. Patten (Eds.), Guelph, Ontario: Canadian Association for Publishing in Philosophy: 91-118.

12. Clarke. S. 2001. Informed Consent in Medicine in Comparison with Consent in Other Areas of Human Activity. Southern Journal of Philosophy 39: 169-187.

13. Hunter, D. and Pierscionek, B. K. 2007. Children, Gillick Competency and Consent for Involvement in Research. Journal of Medical Ethics 33: 659-662.

14. Drane, J. 1985. The Many Faces of Competency. Hastings Center Report 15, 2: 17-21.

15. Culver, C. M. and Gert, B. 1990. The Inadequacy of Incompetence. The Milbank Quarterly 68, 4: 619643: 635 .

16. Buchanan, A. 2006. Competency to Stand trial and the Seriousness of the Charge. J. Am. Acad. Psychiatry Law 34: 458-65.

17. Gold, J. I. and Shadlen, M. N. 2007. The Neural Basis of Decision Making. Annual Review of Neuroscience 30: 535-74.

18. Libet, B., Gleason, C. A., Wright E. W. and Pearl, D. K. 1983. Time of Conscious Intention to Act in Relation to Onset of Cerebral Activities (Readiness-Potential): The Unconscious Initiation of a Freely Voluntary Act. Brain 106: 623-642 
19. Reddi, B. A., Asrress, K. N. and Carpenter. R. H. 2003. Accuracy, Information and Response Time in a Saccadic Decision Task, Journal of Neurophysiology 90: 3538-46.

20. Domasio. A. 1994. Descartes’ Error: Emotion, Reason and the Human Brain. London: Picador.

21. Beauregard, M., Levesque, J. and Bourgouin, P. 2001. Neural Correlates of Conscious Self-Regulation of Emotion, Journal of Neuroscience 21: 165: 1-6.

22. Padoa-Schioppa C. and Assad. J. A. 2006. Neurons in the Orbitofrontal Cortex Encode Economic Value. Nature 441: 223-26

23. Yeung, N. and Sanfrey, A. G. 2004. Independent Coding of Reward Magnitude and Valence in the Human Brain. Journal of Neuroscience 24: 6258-64.

24. Northoff, G. 2006. Neuroscience of Decision Making and Informed Consent: an Investigation in Neuroethics. Journal of Medical Ethics 32: 70-73.

25. Palmer B. W. and Savla. G. N. 2007. The Association of Specific Neuropsychological Deficits with Capacity to Consent to Research or Treatment. Journal of the International Neuropsychological Society 13: $1047-1059$

26. Candilis, P. J. and Lidz, C. W. 2010. Advances in Informed Consent Research. In The Ethics of Consent; Theory and Practice. F. G. Miller and A. Wertheimer (eds.) New York: Oxford University Press: $329-346$.

27. Hansson. S. O. 2001. Coping with the Unpredictable Effects of Future Technologies. Philosophy and Technology 24, 2: 137-149.

28. Jotterand, F. McClintock, S. M., Alexander, A. A., and Husain. M. M. 2010. Ethics and Informed Consent of Vagus Nerve Stimulation (VNS) for Patients with Treatment-Resistant Depression (TRD). Neuroethics, 3: 13-22. 
29. Farah, M. J., Smith, M. E., Gawagu, C., Lindsell, D. and Foster. D. 2008. Brain Imaging and Brain Privacy: A Realistic Concern? Journal of Cognitive Neuroscience. 21, 1: 119-127.

30. Räikkä, J. 2010. Brain Imaging and Privacy. Neuroethics, 3, 5-12.

31. Goffman, E. 1982. The Presentation of Self in Everyday Life. Reading: Penguin.

32. Nissenbaum. H. 2004. Privacy as Contextual Integrity. Washington Law Review, 79: 119-158.

33. Kalra, D., Gertz, R., Singleton, P. and Inskip, H. M. 2006. Confidentiality of Personal Health Information Used for Research. British Medical Journal. 333, 196-198.

34. Frederick, S. 2002. Automated Choice Heuristics. In Heuristics and Biases: the Psychology of Intuitive Judgment .T. Gilovich, D. Griffin and D. Kahneman (eds.). Cambridge: Cambridge University Press: 589-558.

35. Bostrom, N. and Sandberg, A. 2009. Cognitive Enhancement: Methods, Ethics, Regulatory Challenges, Science and Engineering Ethics, 15: 311-41.

36. Savulescu, J. and Bostrom, N. (eds). 2009. Human Enhancement. Oxford: Oxford University Press.

37. Savulescu, J., ter Meelen, R. and Kahane, G. (eds). 2011. Enhancing Human Capacities. Oxford: WileyBlackwell. 


\section{University Library}

\section{- M M N E R VA A gateway to Melbourne's research publications}

Minerva Access is the Institutional Repository of The University of Melbourne

Author/s:

Clarke, S

Title:

The Neuroscience of Decision Making and Our Standards for Assessing Competence to Consent

Date:

2013-04

Citation:

Clarke, S. (2013). The Neuroscience of Decision Making and Our Standards for Assessing Competence to Consent. Neuroethics, 6 (1), pp.189-196. https://doi.org/10.1007/ s12152-011-9144-2.

Persistent Link:

http://hdl.handle.net/11343/282611 